\title{
Correlation of Plasma Soluble Fas Ligand Levels with Severe Anaemia in Gabonese and Indian Plasmodium falciparum Malaria Patients
}

\author{
Vincent Guiyedi ${ }^{1-2, \#}$, Prakash Deshpande ${ }^{3, \#}$, Constantin Fesel ${ }^{4}$, Rajendra Jain ${ }^{5}$, Arnaud Dzeing-Ella ${ }^{6}$, \\ Pierre-André Cazenave ${ }^{1}$, Maryvonne Kombila ${ }^{2}$, Gyan Chandra Mishra ${ }^{3}$ and Sylviane Pied ${ }^{1-4, *}$
}

\author{
${ }^{1}$ Unité d'Immunophysiopathologie Infectieuse, URA CNRS 1961, Institut Pasteur, Paris, France; ${ }^{2}$ Département de \\ Parasitologie-Mycologie-Médecine Tropicale, Faculté de Médecine et des Sciences de la Santé, Libreville, Gabon; \\ ${ }^{3}$ National Centre for Cell Science, Pune, Maharashtra, India; ${ }^{4}$ Instituto Gulbenkian de Ciência, Oeiras, Portugal; \\ ${ }^{5}$ K.T.S.Hospital, Dist. Gondia, Maharashtra, India and ${ }^{6}$ Département de Médecine Interne, Centre Hospitalier de \\ Libreville Gabon
}

\begin{abstract}
A comparative analysis of the plasma concentrations of soluble Fas-L (sFas-L) and their correlation with hemoglobin levels and malaria severity was carried out in cohorts of P. falciparum-infected patients from Gabon and India. Young patients from Gabon had plasma levels of sFas-L that increased with disease severity. In contrast, in Indian adults plasmatic sFas-L levels were more elevated in UM and SNCM than in CM. In both Gabonese and Indian subjects, sFas-L concentrations were negatively correlated with haemoglobin rates, which were lower in SNCM than in UM or CM in both populations. We also observed a positive correlation between the level of plasmatic sFas-L with the level of circulating IL-2 receptor in the Indian patients. All these observations suggest a paradoxical role of sFas-L in CM pathogenesis when comparing Gabonese and Indian patients, while a similar role was found to be associated with severe anaemia.
\end{abstract}

Keys Words: sFasL, P. falciparum, severe malaria.

\section{INTRODUCTION}

Malaria is still a major public health problem in tropical countries, especially in Sub-Saharan Africa and southern Asia. About 1,7 million of the two billion people living in these endemic areas die every year from complications induced by $P$. falciparum infection, such as severe anaemia and cerebral malaria (CM), often associated with hypoglycemia or hyperlactatemia [1]. CM mostly affects children under 5 years of age in African countries, non immune or immunocompromised individuals, pregnant women and adult in India [2]. CM is a complex syndrome that, in addition to other biological processes, involves the sequestration of mature forms of parasitized red blood cells (pRBCs) into brain microvessels and the host's immune responses. Parasite sequestration is a phenomenon that elicits molecular interactions between parasite ligands located on the surface of pRBCs and receptors expressed on the surface of endothelial cells [3]. In spite of the description of these phenomenona, the key mechanisms underlying fatal manifestations of $P$. falciparum malaria still need to be investigated. Several lines of evidence support a role for pathways involving FasFas ligand (Fas-L) interactions in immunophysiopathological events leading to CM $[4,5]$. This is supported by the observation that Lpr, Gld, Fas and Fas ligand deficient mice infected with $P$. berghei ANKA, although shown to develop

\footnotetext{
*Address correspondence to this author at the Unité INSERM U547, Institut Pasteur de Lille, 1 rue du Pr A. Calmette, 59019 Lille, France; Tel: (33)3 20 8778 02; Fax: (33)3 208778 88; E-mail: sylviane.pied@pasteur-lille.fr "Both authors contributed equally to this work.
}

cerebral pathology to some extent, are protected from $\mathrm{CM}$ [5].

Fas-L belongs to the protein family that includes TNF $\alpha$ and related cytokines [6]. Fas is expressed on target cells and Fas- $\mathrm{L}$ on activated effector cells either on cell membranes (mFas-L) or in soluble form (sFas-L) [7]. sFas-L is derived from $\mathrm{mFas}-\mathrm{L}$ through cleavage by metalloproteases [8]. Interaction of the receptor with its ligand is one of the mechanisms used to kill target cells by apoptosis, a process in which a cell dies in a controlled and programmed manner in response to stimulation [9]. Apoptosis is also an efficient regulatory mechanism that controls $\mathrm{T}$ cell numbers during homeostasis as well as during immunosuppression [10]. As an example, sFas-L can kill activated $\mathrm{T}$ cells during antigen activation-induced cell death [11]. However, systemic tissue damage can also be due to elevated levels of cytotoxic sFas-L [12].

It is known in humans that Plasmodium infection induces rapid elevation of sFas-L [13, 14]. The increase in serum levels of sFasL has been associated with severe anaemia during $P$. falciparum malaria or hepatic dysfunction during $P$. vivax infection $[14,15]$. Moreover, a marked increase in the serum concentration of sFas-L correlated with significant lymphopenia before antimalarial treatment in P. falciparuminfected patients $[13,16]$. These levels significantly declined during the course of infection. These observations suggest that severe lymphopenia could be due, in part, to a high apoptosis rate of T lymphocytes [13, 17]. However, whether these phenomena are involved in the pathophysiology of CM remains to be clarified. 
So far, there is no clear information regarding the role of FasL in the pathogenesis of CM in humans. In the present study conducted in two malaria transmission areas, India and Gabon, we investigated whether the Fas-Fas-L-mediated apoptosis pathway is associated with $\mathrm{CM}$ and/or severe anaemia by evaluating the circulating level of sFas-L in patients manifesting different clinical forms of $P$. falcipraum infection. In particular, we wanted to assess if the plasma level of sFAS-L could be used as a marker of gravity.

\section{PATIENTS A-ND METHODS}

\subsection{Study Population}

The present study was carried out in India and Gabon. Patients were included in the study only after informed consent had been obtained from their parents. The ethics committee of the Indian and Gabon Health Office approved of this study. Indian patients were recruited from 2001 to 2003 from different rural hospitals in the Gondia district of Maharashtra State. Patients from Gabon were recruited at the Libreville Hospital Center (LHC) between 1999 and 2002.

Indian and Gabonese patients were assigned to various groups on the basis of World Health Organization guidelines for the definition of uncomplicated and severe malaria. Age of malaria patients ranged from 5 to 75 years and from 4 months to 5 years in the Indian and Gabonese cohort, respectively. These patients were classified into three groups of symptomatic $P$. falciparum malaria: 1) uncomplicated malaria (UM), 2) severe non-cerebral malaria (SNCM) with severe anaemia (haemoglobin level $<5 \mathrm{~g} / \mathrm{dL}$ ) or hypoglycemia (glycemia $<2.2 \mathrm{mmol} / \mathrm{mL}$ ), and 3) cerebral malaria (CM) with a Blantyre Coma Score $\leq 2$ and for adults a Glasgow score $\leq 9$ or one convulsive episode during the 24 hours prior to admission, with a stated post-critical comatose lasting > 15 minutes. In addition, three groups of asymptomatic $P$. falciparum uninfected controls were included in the Indian cohort: ex-CM patients, endemic control patients (EC) and non-endemic control patients (NEC). Blood samples of endemic controls were collected from relatives of patients (brothers/sisters/parents) who accompanied them to the hospital. These individuals were not suffering from malaria for at least two years and were not clinical symptomatic carriers. Non-endemic blood controls were collected from voluntary individuals with no history of malarial disease for 5 years or more. Ex-CM samples were collected from the CM patients group three (3) months later. In the Gabonese cohort, two control groups from the same area of Libreville City was also studied; the uninfected endemic controls (EC), comprising children with $P$. falciparum-negative thick blood smears, and the asymptomatic infected (AI) patients with positive $P$. falciparum thick blood smears. All patients presenting diseases other than malaria were excluded from the study. Parasitemia (thick blood smears) was determined on day 0 , the day of hospitalization using the Lambarene method [18].

Gabonese and Indian patients were treated with quinine, depending on disease severity. The patients with uncomplicated malaria were given oral quinine $(25 \mathrm{mg} / \mathrm{kg} /$ day $)$ for five days; those with severe malaria received intravenous quinine $(25 \mathrm{mg} / \mathrm{kg} / \mathrm{day})$ for five days. Children and adult patients with severe anaemia underwent blood transfusion.

Venous blood (5-10 mL for Indian adults and $2 \mathrm{~mL}$ for Gabonese children patients) from each patient was collected in sterile vacutainers containing EDTA at the day of hospitalization. Plasma was obtained by centrifuging blood samples at $5000 \mathrm{~g}$ for $15 \mathrm{~min}$. Plasma samples were stored at $80^{\circ} \mathrm{C}$ until use.

\subsection{Quantification of sFas-L}

The plasma concentrations of sFasL were quantified for each patient by sandwich-type ELISA using a kit (Duoset ${ }^{R}$ ELISA Development System, R\&D System, France). 96 microwell plates (Reacti-Bind 96 EIA Plat.Corn.Not. $100 /$ KKG, Pierce) were coated with $5 \mu \mathrm{g} / \mathrm{mL}$ of mouse antihuman Fas Ligand antibodies overnight at $4^{\circ} \mathrm{C}$. After washing with PBS-0.05\% Tween $^{\mathrm{R}} 20$, plates were saturated with PBS containing $1 \% \mathrm{BSA}$ in PBS for 1 hour at $37^{\circ} \mathrm{C}$. Plasma samples $(100 \mu \mathrm{L})$ and recombinant Fas Ligand standard (100 $\mu \mathrm{L})$ were added to each coated well and incubated for 2 hours at room temperature. After washing, the plates were incubated with $100 \mu \mathrm{L}$ of biotinylated goat anti human Fas Ligand $(50 \mathrm{ng} / \mathrm{mL})$ for $2 \mathrm{~h}$ at room temperature. After washing, plates were incubated for 20 minutes at room temperature with $100 \mu \mathrm{L}$ of $1 / 200$ Streptavidin conjugated to Horseradish-Peroxidase. Bound enzyme reactivity was revealed in the dark using $100 \mu \mathrm{L} /$ well of O-Phenylenediamine (OPD) substrate in $0.05 \mathrm{M}$ phosphate-citrate buffer, pH5 (SigmaAldrich, France). The peroxidase reaction was stopped after 20 min using $50 \mu \mathrm{L}$ of $2 \mathrm{~N} \mathrm{H} 2 \mathrm{SO} 4$ solution. Optical densities were measured at $450 \mathrm{~nm}$ with an ELISA plate reader. Quantities of sFas Ligand in the samples were calculated from a standard curve ranging between 2000-1.95 and $100.10^{3}-10 \mathrm{pg} / \mathrm{mL}$ for Gabonese and Indian sFas-L quantification, between 1000-1.95 and $500.10^{3}-5 \mathrm{pg} / \mathrm{mL}$ for Indian IL2 and sIL-2R measures respectively. Each sample was tested in duplicate.

\subsection{Quantification of IL-2 and Soluble IL-2 Receptor (sIL2-R)}

A sandwich-type ELISA was used to determine IL-2 and IL-2 $\mathrm{R}$ levels in Indian patients using a kit (OptEIA set, Pharmingen, BD Bioscience, France) according to the manufacturer's recommendations.

\subsection{Haemoglobin and Leukocyte Quantification}

Haemoglobin concentrations were measured in both Gabonese and Indian patients using a coulter (STKS, Coulter counter Corporation). Leukocyte numbers were determined for patients from Gabon.

\subsection{Statistical Analysis}

All statistics were performed by nonparametric methods. Qualitative comparisons were performed using the MannWhitney rank sum test. Correlations were assessed by Spearman rank correlation. P-values below 0.05 were considered significant.

\section{RESULTS}

\subsection{General Characteristics of the Study Population}

A total of 114 patients from Gabon (Table 1) and 104 patients from India (Table 2) were studied. The numbers of controls or patients developing malaria were equally distributed between the two cohorts: UM ( $n=37$ versus 33 , respec- 
Table 1. sFas-L Concentrations, Leukocytes Numbers and Haemoglobinemia in Respect to Clinical Groups in Gabonese Patients from

\begin{tabular}{|c|c|c|c|c|c|c|}
\hline & $\begin{array}{c}\text { EC } \\
(\mathbf{N}=13)\end{array}$ & $\begin{array}{c}\text { AI } \\
(\mathbf{N}=11)\end{array}$ & $\begin{array}{c}\text { UM } \\
(\mathbf{N}=37)\end{array}$ & $\begin{array}{l}\text { SNCM } \\
(\mathrm{N}=29)\end{array}$ & $\begin{array}{c}\mathrm{CM} \\
(\mathrm{N}=\mathbf{2 4})\end{array}$ & $p$ value \\
\hline $\begin{array}{c}\text { Leukocytes number } / \mathbf{m m}^{3} \\
\text { median } \\
{[\text { extrems }]}\end{array}$ & - & - & $\begin{array}{c}8300^{\mathrm{a}} \\
{[3800-11600]}\end{array}$ & $\begin{array}{c}9700^{a} \\
{[3300-31800]}\end{array}$ & $\begin{array}{c}15100^{\mathrm{a}} \\
{[7000-47900]}\end{array}$ & $\mathrm{a}: 0.02$ \\
\hline $\begin{array}{c}\text { Haemoglobinemia }(\mathrm{g} / \mathrm{L}) \\
\text { median } \\
{[\text { extrems] }}\end{array}$ & - & - & $\begin{array}{c}8^{a} \\
{[7-13]}\end{array}$ & $\begin{array}{c}5^{\mathrm{ab}} \\
{[3-11]}\end{array}$ & $\begin{array}{c}13^{\mathrm{b}} \\
{[2-11]}\end{array}$ & $\begin{array}{l}\mathrm{a}: 0.001 \\
\mathrm{~b}: 0.02\end{array}$ \\
\hline
\end{tabular}

EC: uninfected endemic controls, AI: asyptomatic infected patient, UM: uncomplicated malaria, SNCM: severe non cerebral malaria, CM: cerebral malaria.

Table 2. sFas-L, IL-2, sIL-2R Concentrations and Haemoglobinemia in Respect to Clinical Groups in Indian Patients

\begin{tabular}{|c|c|c|c|c|c|c|c|}
\hline & $\begin{array}{l}\text { NEC } \\
(N=9)\end{array}$ & $\begin{array}{c}\text { EC } \\
(N=14)\end{array}$ & $\begin{array}{c}\text { UM } \\
(\mathbf{N}=\mathbf{3 3})\end{array}$ & $\begin{array}{l}\text { SNCM } \\
(N=14)\end{array}$ & $\begin{array}{c}\mathrm{CM} \\
(\mathrm{N}=26)\end{array}$ & $\begin{array}{c}\begin{array}{c}\text { Ex-CM } \\
(N=8)\end{array}\end{array}$ & $p$ value \\
\hline $\begin{array}{c}\text { sFas-L levels }(\mathrm{pg} / \mathrm{mL}) \\
\text { median } \\
{[\text { extrems] }}\end{array}$ & $\begin{array}{c}50^{\mathrm{c}} \\
{[20-280]}\end{array}$ & $\begin{array}{c}1000^{\mathrm{b}} \\
{[100-1600]}\end{array}$ & $\begin{array}{c}23000^{\mathrm{ab}} \\
{\left[10-36.10^{3}\right]}\end{array}$ & $\begin{array}{c}55000^{\mathrm{ab}} \\
{\left[36-69.10^{3}\right]}\end{array}$ & $\begin{array}{c}1700^{\mathrm{ac}} \\
{[1200-2000]}\end{array}$ & $\begin{array}{c}1240 \\
{[1000-1600]}\end{array}$ & $\begin{array}{l}\mathrm{a}:<0.001 \\
\mathrm{~b}:<0.001 \\
\mathrm{c}:<0.001\end{array}$ \\
\hline $\begin{array}{c}\text { IL-2 levels }(\mathrm{pg} / \mathrm{mL}) \\
\text { median } \\
{[\text { extrems] }}\end{array}$ & $\begin{array}{c}23 \\
{[10-36]}\end{array}$ & $\begin{array}{c}51 \\
{[31-69]}\end{array}$ & $\begin{array}{c}690^{\mathrm{ab}} \\
{[493-896]}\end{array}$ & $\begin{array}{c}553^{\mathrm{a}} \\
{[493-720]}\end{array}$ & $\begin{array}{c}322^{\mathrm{bc}} \\
{[150-450]}\end{array}$ & $\begin{array}{c}63^{\mathrm{c}} \\
{[50-92]}\end{array}$ & $\begin{array}{l}\mathrm{a}: 0.003 \\
\mathrm{~b}:<0.001 \\
\mathrm{c}:<0.001\end{array}$ \\
\hline $\begin{array}{c}\text { sIL-2R levels }(\mathrm{pg} / \mathrm{mL}) \\
\text { median } \\
{[\text { extrems] }}\end{array}$ & $\begin{array}{c}26.10^{3 \mathrm{~b}} \\
{\left[15-160.10^{3}\right]}\end{array}$ & $\begin{array}{c}64.10^{3 \mathrm{~b}} \\
{\left[44-88.10^{3}\right]}\end{array}$ & $\begin{array}{c}117.10^{3 \mathrm{ab}} \\
{\left[83-146.10^{3}\right]}\end{array}$ & $\begin{array}{c}193.10^{3 \mathrm{a}} \\
{\left[119-221.10^{3}\right]}\end{array}$ & $\begin{array}{c}185.10^{3 \mathrm{a}} \\
{\left[138-290.10^{3}\right]}\end{array}$ & $\begin{array}{c}60.10^{3} \\
{\left[55-70.10^{3}\right]}\end{array}$ & $\begin{array}{l}\mathrm{a}:<0.001 \\
\mathrm{~b}:<0.001\end{array}$ \\
\hline $\begin{array}{c}\text { Haemoglobinemia }(\mathrm{g} / \mathrm{L}) \\
\text { median } \\
{[\text { extrems }]}\end{array}$ & - & - & $\begin{array}{c}11^{\mathrm{a}} \\
{[7-13]}\end{array}$ & $\begin{array}{c}7^{\mathrm{ab}} \\
{[6-10]}\end{array}$ & $\begin{array}{c}11^{\mathrm{b}} \\
{[8-13]}\end{array}$ & - & $\begin{array}{l}a:<0.001 \\
b:<0.001\end{array}$ \\
\hline
\end{tabular}

EC: uninfected endemic controls, NEC: non endemic control, UM: uncomplicated malaria, SNCM: severe non cerebral malaria, CM: cerebral malaria, Ex-CM: Ex cerebral malaria.

tively), SNCM ( $\mathrm{n}=29$ versus 14$), \mathrm{CM}(\mathrm{n}=24$ versus 26$), \mathrm{EC}$ $(n=13$ versus $n=14)$, NEC $(n=9)$, AI $(n=11)$ and ex-CM patients $(n=8)$. Ages of malaria patients ranged from 0.3 to 10 years in Gabonese and from 2.2 to 75 years in Indian patients. There was no significant difference in parasitemia and mean age between the different Indian and Gabonese patient cohorts, although the mean age of individuals was significantly higher in India than in Gabon.

\subsection{Analysis of the Relationship between Levels of Circu- lating sFasL and Malaria Clinical Phenotype in Indian and Gabonese $P$. falciparum-Infected Patients}

Concentrations of sFas-L were determined in patient plasma of Gabonese and Indian cohorts. In Gabonese patients, plasma levels of sFas-L were enhanced in parallel with disease severity, and were highest in the CM group (median: 830, range 245-2108 pg/mL), followed by SNCM (median 658, range 160-1256 pg/mL) and UM (median 335, range 2-569 $\mathrm{pg} / \mathrm{mL}$ ), while $\mathrm{AI}$ and EC groups exhibited median levels of 38 and $64 \mathrm{pg} / \mathrm{mL}$, respectively, ranging between 1.95 and $265 \mathrm{pg} / \mathrm{mL}$ (Table 1). The plasma concentrations of sFas-L were significantly higher in CM than in SNCM $[p=0.04]$. Similar results were observed when comparing sFas-L amount in patients developing severe malaria (including $\mathrm{CM}$ and $\mathrm{SNCM}$ ) with the UM group [p<0.001], and in UM compared to the two control groups (EC and AI) $[p<0.001]$. No significant difference was observed between $\mathrm{EC}$ and $\mathrm{AI}$.

It is noteworthy that plasma sFas- $\mathrm{L}$ concentrations in respect to malaria severity in Indian patients were different from results observed in Gabonese cohort. Thus, Indian patients developing malaria without cerebral attacks, including UM (median 23000, range 13000-36000 pg/mL) or SNCM (median 55000, range 36000-69000 pg/mL) presented higher concentrations of sFas-L when compared to CM (median 1700 , range $1200-2000 \mathrm{pg} / \mathrm{mL}$ ) [p<0.001 respectively] or EC (median 1000, range 100-1600 pg/mL) [p<0.001] (Table 2). 
However, the slight absolute increase in $\mathrm{CM}$ was significant when compared to NEC ( $\mathrm{p}<0.001)$. Thus, even if the average concentration of sFas-L in plasma was significantly higher in Indian compared to Gabonese CM patients [ $\mathrm{p}<0.001]$, these two populations exhibit opposite behavior in their sFas-L production during disease outcome. In addition, no overall or groupwise correlation of sFas-L plasma levels with age or parasite load was established in the two cohorts. In conclusion, while the highest levels of sFas-L were detected in Gabonese CM, results clearly show a lake of association of high plasma concentrations of this cytokine with $\mathrm{CM}$ in patients from India.

\subsection{A High Concentration of Circulating sFas-L Corre- lates with Low Haemoglobinemia and Malaria Severity}

Haemoglobinemia was quantified for all groups of Gabonese and Indian malaria patients (UM, SNCM, CM). Anaemia was more important in Gabonese children than in adult Indian patients (Table $\mathbf{1}$ and Table 2, respectively). As expected, SNCM patients showed the lowest haemoglobin concentrations in the two populations and were not significantly different between UM and CM but were significantly higher in UM and CM than in SNCM $[\mathrm{p}<0.001$, and $\mathrm{p}=0.02$, respectively] in the Gabonese population (Table 1). Comparatively, haemoglobin concentrations were also significantly higher in UM and CM than in SNCM $[\mathrm{p}<0.001$, respectively] (Table 2) in the Indian patients. The difference between UM and CM was not significant.

When analysing each group of patients separately, no correlation was found between sFas-L and haemoglobin levels in UM, SNCM or CM, although a significant negative correlation was observed between sFas-L and haemoglobin concentrations for all patients of the two cohorts [Spearman $\mathrm{R}=-0.28, \mathrm{p}=0.006$ (Fig. 1A) and $\mathrm{R}=-0.56, \mathrm{p}=0.0002$ (Fig. 1B), respectively in Gabon and Indian cohort].
From these observations we can conclude that during malaria, independent of disease severity, a high level of circulating sFas-L is associated with low haemoglobin rate.

3.4. Correlation between Plasma Levels of sFas-L, IL-2, and SIL-2R in Indian Patients

We also quantified the level of IL-2 and sIL-2R for each patient in the Indian cohorts. Patients with high IL-2 concentrations were distributed throughout the three diseased groups (including UM, SNCM and CM) (Table 2). IL-2 concentrations were significantly higher in UM than in SNCM and $\mathrm{CM}[\mathrm{p}=0.003$ and $\mathrm{p}<0.001]$, and were significantly higher in all three diseased groups than in the control patient groups (including NEC, EC and Ex-CM group) [p<0.0001]. In contrast to IL-2, plasma sIL-2R was significantly higher in patients developing severe malaria (SNCM and CM) compared to the UM group [p<0.001 respectively] (Table 2). Plasma sIL-2R concentrations were significantly higher in UM than in controls (NEC and EC) [p<0.001]. In patients developing disease (UM, SNCM and CM), there was a significant negative correlation of IL-2 with sIL-2R $[R=-0.64$, $\mathrm{p}<0.001]$ (data not shown).

It is noteworthy that Indian CM patients presented low levels of sFas- $\mathrm{L}$ in the plasma. In the other groups of patients (NEC, EC, UM and SNCM), sFas-L plasma concentrations showed an extremely high positive correlation with plasma concentrations of sIL-2R [Spearman $\mathrm{R}=+0.90, \mathrm{p}<0.001$ ] (Fig. 2A).

\subsection{Correlation between Plasma Levels of sFas-L and Leukocyte Numbers in Patients from Gabon}

Leukocyte number were determined for patients included in the Gabonese groups. We found a significantly higher number of leukocytes in CM than in both UM and SNCM $[p=0.002]$ (Table 1). In SNCM patients that developed

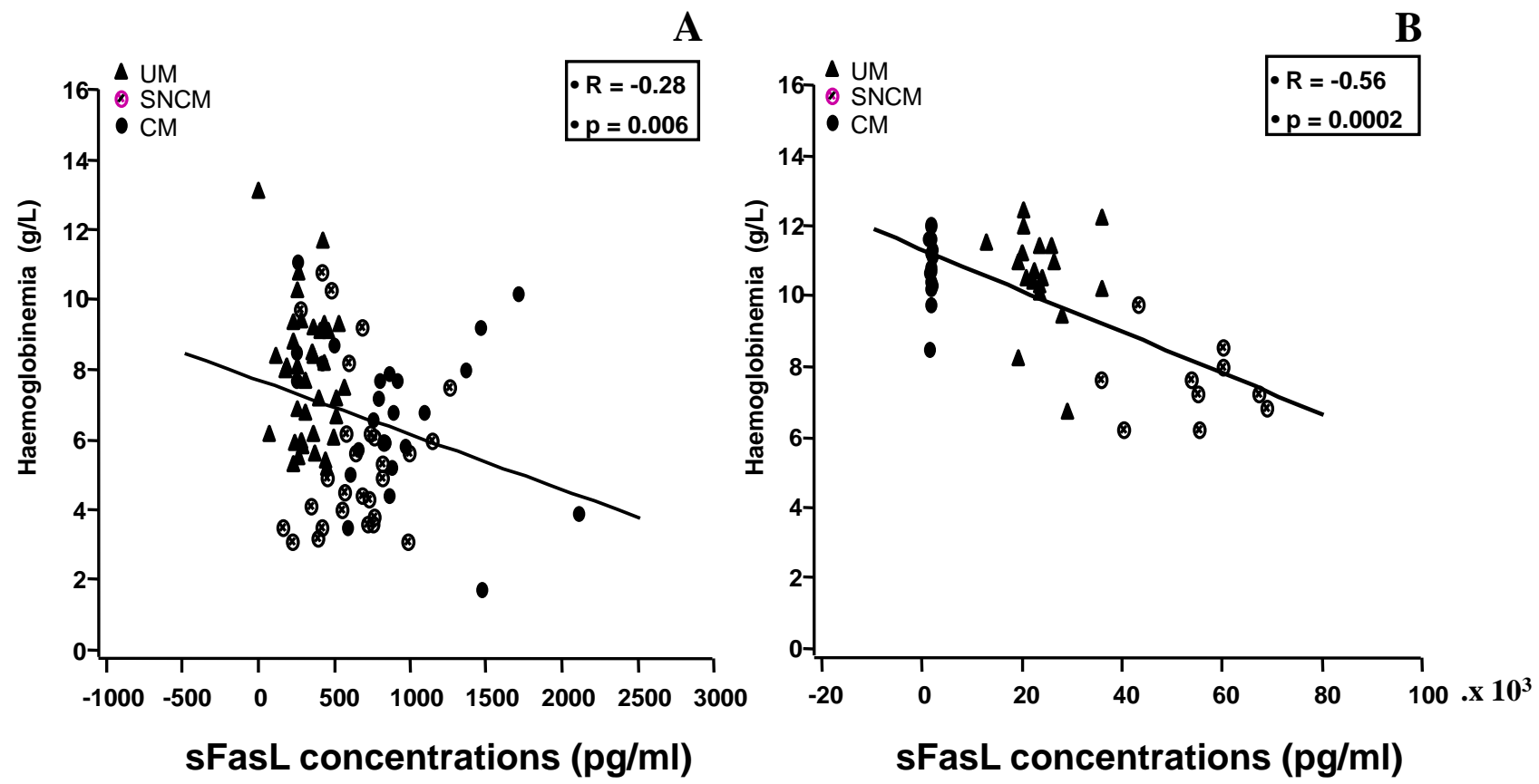

Fig. (1). Correlation between plasma sFas-L and haemoglobin concentrations in Gabonese (A) and Indian (B) cohort. UM: uncomplicated malaria, SNCM: severe non cerebral malaria, CM: cerebral malaria. 

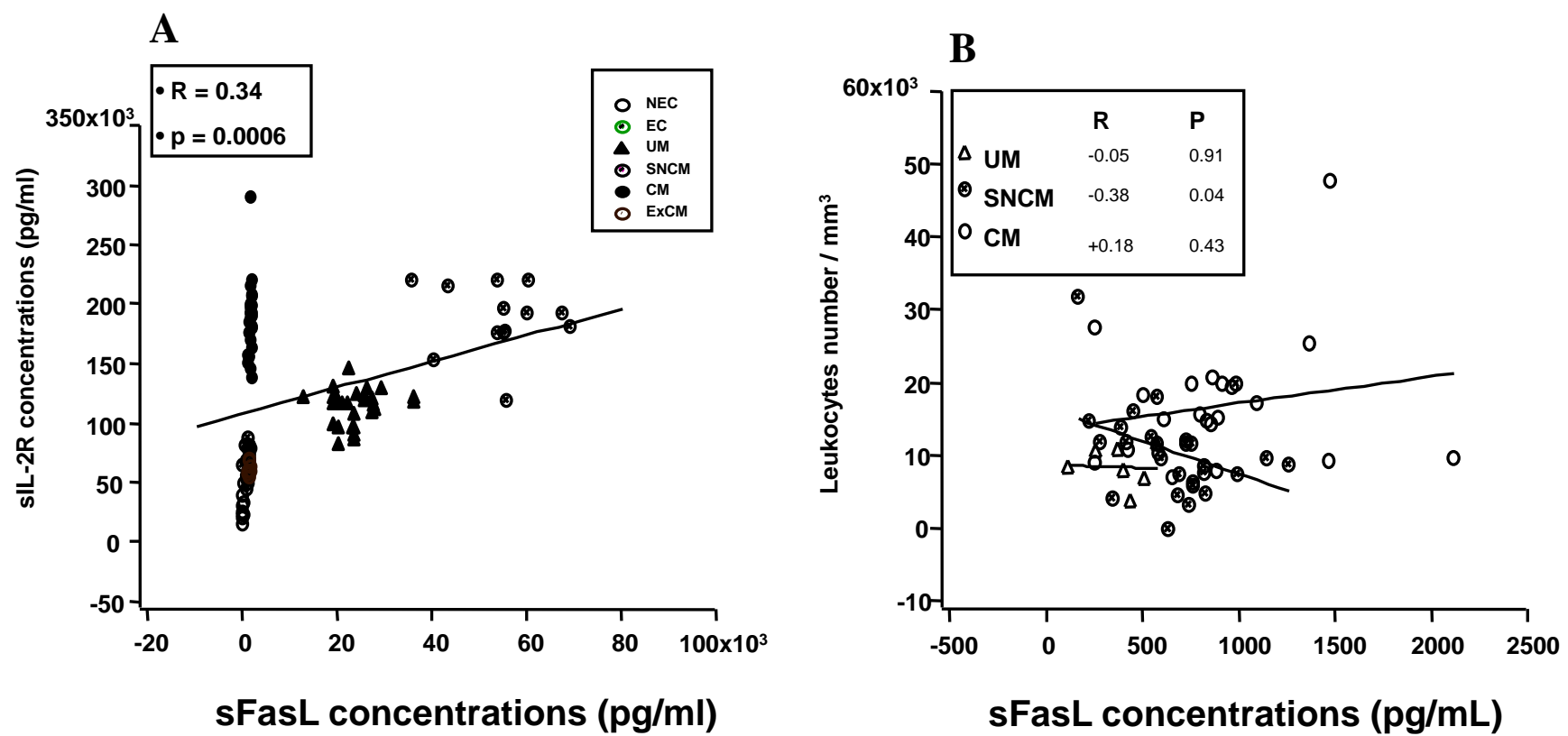

Fig. (2). (A).Correlation between sFas-L and sIL-2R in Indian cohort. (B). Correlation between sFas-L and leukocytes number in Gabonese patients. EC: endemic control, NEC: non endemic control, UM: uncomplicated malaria, SNCM: severe non cerebral malaria, CM: cerebral malaria, Ex-CM: Ex cerebral malaria.

anaemia (haemoglobin rate $<10 \mathrm{~g} / \mathrm{dl}$ ), we observed a significant negative correlation between sFas-L concentrations and leukocyte number [Spearman $\mathrm{R}=-0.40, \mathrm{p}=0.04]$. In the $\mathrm{CM}$ group, this correlation was positive but not significant (Fig. 2B). This observation suggests that the decrease in leukocyte number could be associated with apoptotic mechanisms.

\section{DISCUSSION}

The precise of events that characterizes severe anaemia and $\mathrm{CM}$, the two major complications of a $P$. falciparum infection, responsible for the majority of malaria-associated deaths remain undefined $[1,2]$. The Fas-FasL system is a double-edged sword in that both diminution and enhancement of this system have pathogenic effects [19]. To better understand the role of Fas-FasL-mediated apoptosis during the outcome of a Plasmodium infection in humans as well as in the search for biological markers allowing for better definition of malaria phenotypes, we investigated the role of sFas-L by quantifying its level in the plasma of Indian and Gabonese $P$. falciparum-infected patients developing asymptomatic, uncomplicated, severe non-cerebral and CM. We also analysed whether the level of circulating sFasL correlated with haemoglobin rates, IL-2 and IL-2R levels, peripheral leucocytes number could contribute to disease severity.

Our data show a significant increase in plasma sFas-L levels in association with the degree of disease severity, particularly in CM Gabonese patients. These levels were lowest in Indian $\mathrm{CM}$ patients compared to diseased groups without cerebral attacks. In addition, sFas-L concentrations were negatively correlated with haemoglobin concentrations both in Indian and Gabonese patients. To our knowledge, the first description of an association between sFas-L and anaemia in children developing malaria was performed in Gabon three years ago, in Lambaréné, a town located in the center of the country [15]. Our observations in children from Libreville, situated in the East of the same country (where malaria is hyper-endemic), and adults from India, a different continent were malaria is hypo-endemic, confirm the hypothesis of the existence of an apoptotic Fas-L-mediated mechanism during the induction and/or aggravation of anaemia during $P$. falciparum malaria. However, the implication of this sFas-L-mediated mechanism in the destruction of red blood cells during malaria needs to be clarified, although a role for apoptosis has been suggested to be important for the regulation of erythropoesis [20]. Multiple myeloma is a neoplastic disease of the hematopoietic system characterized by the presence of anemia, where expression of Fas is detected on the cell surface membrane of erythroblasts at all developmental stages, whereas sFas-L is present only on more mature erythroblasts. Recent findings also indicate an important role for sFas-L molecules in the pathogenesis of anemia observed in multiple myeloma patients $[21,22]$. FasL molecules are involved in inducing apoptosis of erythroid cells. Similarly, the increase of Fas expression on hematopoietic progenitors during aplastic anemia suggests that the Fas/Fas ligand (FasL) system plays a key role in the formation of severe pancytopenia [23, 24].

It has been demonstrated that $P$. falciparum-infected erythrocytes have the capacity to induce caspase activation and apoptosis of human endothelial cells during malaria [25]. Because $P$. falciparum has the capacity to infect young and mature red blood cells, and young erythrocytes express Fas or Fas-L molecules [20, 23], the destruction of infected and non-infected non-mature red blood cells expressing these molecules by apoptotic mechanisms could be a mechanism responsible for the severe anaemia observed during infection.

To date, a very limited number of studies addressed the role of sFas-L in severe malaria pathogenesis. Published observations implicate a role of the Fas/Fas-L pathway in apoptosis associated with lymphopenia during $P$. falciparum uncomplicated [13] and severe anaemia [15]. It has been 
suggested that activated $\mathrm{T}$ cells persist before and after initiation of therapy during $P$. falciparum malaria with elevated proportion of apoptotic $\mathrm{T}$ cells to re-establish immunological homeostasis [26]. However, this high proportion of apoptotic cells could contribute to the low number of $\mathrm{T}$ cells in the peripheral circulation during active disease [26]. It is noteworthy that no lymphopenia was detected in Gabonese CM patients who exhibited the highest levels of sFas-L, which was positively correlated with high leukocyte number. These results are similar to those observed during experimental $\mathrm{CM}$ induced in C57BL/6 or B10D2 mice by P. berghei ANKA. In these two experimental models, $\mathrm{CM}$ has been associated with a selective expansion and migration of $\mathrm{CD}^{+}$and $\mathrm{CD}^{+}$ $\mathrm{T}$ cells in the brain of mice developing the cerebral syndrome [27, 28].

The first observations suggesting a potential role of sFas$\mathrm{L}$ in severe and particularly $\mathrm{CM}$ pathogenesis were from experimental models in which profound T-lymphopenia due to Fas-derived apoptosis has been found to play a role in fatal malaria induced by $P$. coatneyi in macaques [17]. It has been shown that levels of Fas/Fas-L mRNA and protein expression increased in the brain of mice with CM [5]. In addition, Fas deficient (Lpr) or Fas ligand deficient (Gld) mice are protected from fatal $\mathrm{CM}$ induced by $P$. berge $i$ ANKA [5] and the incidence of CM in Fas/Fas-L-deficient mutant mice was decreased compared to wild-type controls $[4,5]$. However, our results also suggest that the pathogenesis of CM could be associated with inhibition of apoptotic mechanisms mediated by sFas-L molecules in Gabonese patients.

The observed discrepancy between Gabonese and Indian CM patients who had levels of sFas-L comparable to their respective non-infected controls is surprising. Furthermore, the small amounts of sFas-L found in CM patients from India did not correlate with plasma concentrations of IL-2R as observed for SNCM and UM. The increase of sIL-2R concentrations in P. falciparum malaria patients [29-31] and particularly in patients developing severe anaemia has been already reported [32]. In the study performed by EKP Riccio et $a l$, a positive correlation between sIL-2R and apoptosis was observed in the subgroup with an increased percentage of apoptotic cells. Therefore, this increase in apoptosis levels is not a universal phenomena, and apoptosis events could be fluctuating in the same individual during the progression of the disease [30]. However, in our study, differences in sFasL secretion between SNCM, UM and CM in Indian patients could not be explained by individual particularity because all CM patients secreted very low levels of sFas-L. This suggests that the pathogenesis of CM in the Indian population could be associated with immune regulations that lead to a reduction in sFas-L production. An alternative hypothesis could be that, in contrast to severe anaemia, Fas-mediated apoptosis does not play an essential role in human CM and its contribution may vary substantially with undefined environmental or genetic factor differences between our two study populations.

A good example of the influence of genetic factors on levels of sFas-L secretion and its implication in disease outcome was indirectly addressed by our results demonstrating high concentrations of sFas-L in all Indian patients independently of the diseased group when compared to patients from Gabon. The possibility that genetic factors may control susceptibility to cell apoptosis mediated by sFas-L molecules was suggested in the experimental model of malaria in macaques [17].

In conclusion, apoptosis of red blood cells could be one of the mechanisms implicated in the induction and/or the aggravation of anaemia associated with $P$. falciparum infection. Observation of this phenomenon in three different populations (the one described by Issifou et al. and this paper) is in concordance with this hypothesis. sFas-L production and apoptosis mechanisms could be implicated in CM pathogenesis, with different mechanisms who could depend on genetic background of the population studied, age and potentially the immunological history of the patients.

\section{ACKNOWLEDGMENTS}

We thank F. Mbadinga, M. Ekoumembia† and N. Obiang for their assistance in the collection of plasma samples. We also thank U. B. for critical reading of the manuscript.

We thank the Indo-French Centre for Promotion of Advanced Research (IFCPAR), New Delhi, India for providing financial assistance for the project (No.2103-3). This work was also supported by the PAL+ program of the French Ministry of Research and by the CNRS-IGC LEA "Génétique et développement de la tolérance naturelle" program. $\mathrm{C}-\mathrm{F}$ received a post-doctoral fellowship and S-P a visiting scientist fellowship from the Fundaçâo para a Ciência $e$ Tecnologia (Portugal). V-G holds a fellowship from the Agence Universitaire de la Francophonie (AUF).

ABBREVIATIONS
$\mathrm{CM}=$ Cerebral Malaria
$\mathrm{SNCM}=$ Severe Non Cerebral Malaria
$\mathrm{UM}=$ Uncomplicated Malaria
$\mathrm{Ex}-\mathrm{CM}=$ Recovered Cases of Cerebral Malaria
$\mathrm{NEC}=$ Normal Endemic Control
$\mathrm{EC} \quad=\quad$ Endemic Control

\section{REFERENCES}

[1] Dzeing Ella A, Nze Obiang PC, Tchoua R, et al. Severe falciparum malaria in Gabonese children: clinical and laboratory features. Malar J 2005; 9:1.

[2] Breman JG, Egan A, Keusch GT. The intolerable burden of malaria: a new look at the numbers. Am J Trop Med Hyg 2001; 64(1-2 Suppl): 4-7.

[3] Van der Heyde HC, Nolan J, Combes V, Gramaglia I, Grau GE. A unified hypothesis for the genesis of cerebral malaria: sequestration, inflammation and hemostasis leading to microcirculatory dysfunction. Trends Parasitol 2006; 22: 503-8.

[4] Ohno T, Kobayashi F, Nishimura M. Fas has a role in cerebral malaria, but not in proliferation or exclusion of the murine parasite in mice. Immunogenetics 2005; 57: 293-6.

[5] Potter SM, Chan-Ling T, Rosinova E, Ball HJ, Mitchell AJ, Hunt NH. A role for Fas-Fas ligand interactions during the late-stage neuropathological processes of experimental cerebral malaria. J Neuroimmunol 2006; 173: 96-107.

[6] Suda T, Takahashi T, Golstein P, Nagata S. Molecular cloning and expression of the Fas ligand: a noval member of the tumor necrosis factor family. Cell 1993; 75: 1169-78.

[7] Kagi D, Vignaux F, Ledermann B, et al. Fas and perforin pathways as major mechanisms of T cell-mediatedcytotoxicity. Science 1994; 265: 528-30. 
[8] Kayagaki N, Kawasaki A, Ebata T, et al. Metalloproteinasemediated release of human Fas ligand. J Exp Med 1995; 182: 177783.

[9] Blackman MA, Gerhard-Burgert H, Woodland DL, Palmer E, Kappler JW, Marrack P. A role for clonal inactivation in T cell tolerance to Mls-1a. Nature 1990; 345: 540-2.

[10] Raff MC, Barres BA, Burne JF, Coles HS, Ishizaki Y, Jacobson MD. Programmed cell death and the control of cell survival. Philos Trans R Soc Lond B Biol Sci 1994; 345: 265-8.

[11] Dhein J, Walczak H, Baumler C, Debatin KM, Krammer PH. Autocrine T-cell suicide mediated by APO-1/(Fas/CD95). Nature 1995; 373: 438-41.

[12] Tanaka M, Suda T, Yatomi T, Nakamura N, Nagata S. Lethal effect of recombinant human Fas ligand in mice pretreated with Propionibacterium acnes. J Immunol 1997; 158: 2303-9.

[13] Kern P, Dietrich M, Hemmer C, Wellinghausen N. Increased levels of soluble Fas ligand in serum in Plasmodium falciparum malaria. Infect Immun 2000; 68: 3061-3.

[14] Yeom JS, Park SH, Ryu SH, et al. Serum cytokine profiles in patients with Plasmodium vivax malaria: a comparison between those who presented with and without hepatic dysfunction. Trans $\mathrm{R}$ Soc Trop Med Hyg 2003; 97: 687-91.

[15] Issifou S, Mavoungou E, Borrmann S, et al. Severe malarial anaemia associated with increased soluble Fas ligand (sFasL) concentrations in Gabonese children. Eur Cytokine Netw 2003; 14: 23841.

[16] Balde AT, Aribot G, Tall A, Spiegel A, Roussilhon C. Apoptosis modulation in mononuclear cells recovered from individuals exposed to Plasmodium falciparum infection. Parasite Immunol 2000; 22: 307-18.

[17] Matsumoto J, Kawai S, Terao K, et al. Malaria infection induces rapid elevation of the soluble Fas ligand level in serum and subsequent $\mathrm{T}$ lymphocytopenia: possible factors responsible for the differences in susceptibility of two species of Macaca monkeys to Plasmodium coatneyi infection. Infect Immun 2000; 68: 1183-8.

[18] Planche T, Krishna S, Kombila M, et al. Comparison of methods for the rapid laboratory assessment of children with malaria. Am J Trop Med Hyg 2001; 65: 599-602.

[19] Nagata S. Apoptosis by death factor. Cell 1997; 88: 355-65.

[20] De Maria R, Testa U, Luchetti L, et al. Apoptotic role of Fas/Fas ligand system in the regulation of erythropoiesis. Blood 1999; 93 : 796-803.
[21] Grzasko N. Involvement of apoptosis and proinflammatory cytokines in the pathogenesis of anemia in multiple myeloma. Postepy Hig Med Dosw 2004; 58: 364-71.

[22] Silvestris F, Cafforio P, Grinello D, Dammacco F. Upregulation of erythroblast apoptosis by malignant plasma cells: a new pathogenetic mechanism of anemia in multiple myeloma. Rev Clin Exp Hematol 2002; 1: 39-46.

[23] Liu Y, Pop R, Sadegh C, Brugnara C, Haase VH, Socolovsky M. Suppression of Fas-FasL coexpression by erythropoietin mediates erythroblast expansion during the erythropoietic stress response in vivo. Blood 2006; 108: 123-33.

[24] Li W, Fu J, Wang F, Yu G, Wang Y, Zhang X. Distinct overexpression of Fas ligand on $\mathrm{T}$ lymphocytes in aplastic anemia. Cell Mol Immunol 2004; 1: 142-7.

[25] Pino P, Vouldoukis I, Kolb JP, et al. Plasmodium falciparuminfected erythrocyte adhesion induces caspase activation and apoptosis in human endothelial cells. J Infet Dis 2003; 187: 1283-90.

[26] Kemp K, Akanmori BD, Adabayeri V, et al. Cytokine production and apoptosis among $\mathrm{T}$ cells from patients under treatment for Plasmodium falciparum malaria. Clin Exp Immunol 2002; 127(1): 151-7.

[27] Bagot S, Nogueira F, Collette A, et al. Comparative study of brain CD8+ $\mathrm{T}$ cells induced by sporozoites and those induced by bloodstage Plasmodium berghei ANKA involved in the development of cerebral malaria. Infect Immun 2004; 72: 2817-26.

[28] Boubou MI, Collette A, Voegtle D, Mazier D, Cazenave P-A, Pied $\mathrm{S}$. $\mathrm{T}$ cell response in malaria pathogenesis: selective increase in $\mathrm{T}$ cells carrying the TCR V(beta)8 during experimental cerebral malaria. Int Immunol 1999; 11: 1553-62.

[29] Jakobsen PH, Morris-Jones S, Theander TG. Increased plasma levels of soluble IL-2R are associated with severe Plasmodium falciparum malaria. Clin Exp Immunol 1994; 96: 98-103.

[30] Riccio EK, Junior IN, Riccio LR, et al. Malaria associated apoptosis is not significantly correlated with either parasitemia or the number of previous malaria attacks. Parasitol Res 2003; 90: 9-18.

[31] Riley EM, Rowe P, Allen SJ, Greenwood BM. Soluble plasma IL-2 receptors and malaria. Clin Exp Immunol 1993; 91: 495-9.

[32] Akanmori BD, Kurtzhals JA, Goka BQ, et al. Distinct patterns of cytokine regulation in discrete clinical forms of Plasmodium falciparum malaria. Eur Cytokine Netw 2000; 11(1): 113-8.

This is an open access article licensed under the terms of the Creative Commons Attribution Non-Commercial License (http://creativecommons.org/licenses/ by-nc/3.0/) which permits unrestricted, non-commercial use, distribution and reproduction in any medium, provided the work is properly cited. 